implementation to assess impact on drug errors, but there are plans to review this through the local Medicines Management Group.

\section{P 109 IMPLEMENTATION OF A SMALL, PORTABLE, OPIATE CONVERSION GUIDE FOR STAFF IN AN INDEPENDENT HOSPICE}

Laura Bernstein, Laura Nightingale. St Catherine's Hospice, Crawley, England

10.1136/bmjspcare-2014-000654.150

Background Effective and safe titration of medication has a major impact on patient comfort. Confusion surrounding the differing ranges and potencies of opiates and benzodiazepines can result in under- or overdosing of medication leading to inadequate control of symptoms or resultant toxicities respectively. Through staff feedback, teaching sessions and review of drug errors, we identified that staff used differing opioid conversion guides and lacked confidence when converting between benzodiazepines. Staff identified the need for a consistent local approach when converting between different opioids or benzodiazepines using a guide that could be easily accessed across clinical settings.

Aims To provide staff with a conversion table for opioids and benzodiazepines which is evidence-based, cost-effective, replicable and accessible across clinical settings.

To standardise prescribing.

To reduce drug errors related to opioid and benzodiazepine prescribing, conversion $\&$ administration.

Results A small, portable, laminated conversion chart for opioids and benzodiazepines was created to enable consistent conversion between different drugs and between the oral, subcutaneous and transdermal routes. The chart is an identical size to the staff ID badge so that they can be easily attached together to a lanyard for ease of access and use.

Conclusions Unanimously positive feedback was received from staff following the introduction of the conversion chart. In particular, staff value the accessibility and usability of the chart in clinical practice since it has been attached to their lanyard.

It enables less experienced doctors to prescribe accurately and empowers nursing staff to confirm doses prior to administering the medication, using standardised guidance. Following a positive reception, the lanyard-attached conversion chart has now been adopted by the local NHS trust. It is too early following 